\title{
Síndrome de resección anterior baja y su relación con la calidad de vida: aplicación de cuestionario EuroQol-5
}

\author{
Mauricio A. Zambra R. ${ }^{1}$, Günther R. Bocic A ${ }^{1}$., Antonella Sanguinetti M. ${ }^{1}$, \\ Mario A. Abedrapo M. ${ }^{1}$, José L. Llanos B. ${ }^{1}$, Rodrigo Azolas M. ${ }^{1}$, Mauricio J. Díaz B. ${ }^{1}$, \\ Tomas Rodríguez C. ${ }^{1}$, Matías Pruzzo G. ${ }^{1}$ y Felipe Bocic W. ${ }^{2}$
}

'Departamento de Cirugía. Hospital Clínico Universidad de Chile. Santiago, Chile. ${ }^{2}$ CESFAM Federico Puga Borne Servicio de Salud Ñuble. Chillán, Chile.

Recibido 2020-05-20 y aceptado 2020-06-20

Correspondencia a: Dr. Mauricio A. Zambra R mzambrarojas@gmail.com
Low anterior resection syndrome and quality of life: application of EuroQol-5 questionnaire

Introduction: Sphincter-sparing surgeries result in the development of a defecatory dysfunction with different degrees, which is known as low anterior resection syndrome (LARS) and is measured with the LARS Score questionnaire. Objective: To determine the association of the EuroQol-5 questionnaire with the different degrees of LARS Score. Materials and Method: Cross-sectional study, applying the LARS Score and EuroQol-5 questionnaire to patients operated with low and middle rectal cancer, during the period 2004-2017. Demographic analysis and type of surgery are performed. Different statistical tests are used to determine associations between variables, considering a significant $p$ value $<0.05$. Results: 54 patients were surveyed, $62.16 \%$ men, mean age 58.44 years, $37.03 \%$ presented Mayor-LARS. The average quality of life indices for Non-LARS patients is 0.75 , for Minor-LARS is 0.69 and for Mayor-LARS is 0.61 , the difference between indices presents a $p$ value $=0.246 .46 .3 \%$ present problems in habitual activities. LARS Mayor presents an Odd-Ratio of 3.8 and 4.7 for pain/discomfort and anguish/depression respectively. $70 \%$ of patients with LARS Mayor presented SMT and $45 \%$ corresponded to those under 65 years of age. Discussion: There is no statistically significant difference between the quality of life indices according to the LARS Score. Mayor-LARS is more likely to develop some degree of Pain/Discomfort and anguish/depression. The percentage of Mayor-LARS is according to what has been published and the TME is one of the factors with the greatest impact on the development of LARS. Conclusion: The LARS Score is non-significantly related to the quality of life index provided by the EuroQol-5D questionnaire, and there is a tendency to decrease quality of life as the LARS worsens.

Keywords: low anterior resection syndrome; quality of life; rectal cancer.

\section{Resumen}

Introducción: Las cirugías con preservación de esfínter tienen como consecuencia el desarrollo de una disfunción defecatoria con diferentes grados, la cual es conocida como síndrome de resección anterior baja (LARS) y es medida con el cuestionario LARS Score. Objetivo: Determinar la asociación del cuestionario EuroQol-5 (calidad de vida) con los diferentes grados de LARS Score. Materiales y Método: Estudio de tipo transversal, aplicando el cuestionario LARS Score y EuroQol-5 a pacientes operados por cáncer de recto medio y bajo, durante el periodo 2004-2017. Se realiza análisis demográfico y del tipo de cirugía. Para determinar asociaciones entre variables se utilizan diferentes pruebas estadísticas, considerando significativo un valor de $\mathrm{p}<0,05$. Resultados: Se encuestó a 54 pacientes, $62,16 \%$ hombres, promedio de edad 58,44 años, el 37,03\% presentó LARS Mayor. Los índices promedio de calidad de vida para pacientes No LARS es 0,75 , para LARS Menor es 0,69 y para LARS Mayor es 0,61 , la diferencia entre índices presenta un valor $\mathrm{p}=0,246.46,3 \%$ presenta problemas en actividades habituales. LARS Mayor presenta un Odd-Ratio de 3,8 y 4,7 para dolor/malestar y angustia/depresión respectivamente. $70 \%$ de los pacientes con LARS Mayor presentaron resección total del mesorrecto (TME) y el $45 \%$ corresponde a menores de 65 años. Discusión: No existe diferencia estadísticamente significativa entre los índices de calidad de vida 
según LARS Score. LARS Mayor tiene mayor posibilidad de desarrollar algún grado de dolor/malestar y angustia/depresión. El porcentaje de LARS Mayor es acorde a lo publicado y la TME es uno de los factores de mayor impacto en el desarrollo de LARS. Conclusiones: El LARS Score se relaciona de manera no significativa con el índice de calidad de vida entregado por el cuestionario EuroQol-5D, existiendo una tendencia a disminuir la calidad de vida a medida que empeora el LARS.

Palabras clave: síndrome de resección anterior baja; calidad de vida; cáncer de recto.

\section{Introducción}

Actualmente, según GLOBOCAN, el cáncer colorrectal se encuentra dentro de las primeras causas de muerte por cáncer en ambos géneros, con una incidencia alrededor de 20/100.000 habitantes por año. Específicamente el cáncer de recto corresponde a un tercio de los cánceres colorrectales y ha doblado su incidencia en los últimos 20 años ${ }^{1}$.

En las últimas décadas, la resección total del mesorrecto (TME) y la radioquimioterapia neoadyuvante han ayudado a mejorar de forma significativa los resultados oncológicos en los pacientes con tumores rectales ${ }^{2-4}$. Debido a la evolución en las técnicas quirúrgicas y que se aceptan márgenes distales de hasta $1 \mathrm{~cm}$, es que la resección abdominoperineal ha disminuido considerablemente en las últimas décadas, realizándose en la mayoría de los casos, cirugías con preservación de esfínter, sin embargo, dichas cirugías tienen como consecuencia el desarrollo de una disfunción defecatoria con diferentes grados, la cual es conocida como síndrome de resección anterior baja (LARS) y es medida con el cuestionario LARS Score ${ }^{5}$. Los síntomas asociados a LARS van desde aumento en la frecuencia de las deposiciones hasta el grado de incontinencia fecal o disfunción evacuatoria. Sobre el $70 \%$ de los pacientes presentarán algún grado de disfunción defecatoria y un $41 \%$ presentará LARS Mayor 6 .

Diferentes cuestionarios de calidad de vida relacionadas con la atención en salud se han utilizado para asociar los diferentes grados de LARS Score con la calidad de vida de los pacientes, demostrando que ha mayor LARS Score peor calidad de vida ${ }^{7,8}$. El cuestionario de calidad de vida más utilizado es el "European organization for reseach and treatment of cáncer quality life" (EORTC-30), que tiene 30 preguntas, las cuales evalúan 5 dimensiones funcionales de la vida, 8 síntomas y un aspecto económico?.

Por lo anterior, es que nos planteamos como objetivo principal determinar la asociación de un cuestionario de calidad de vida más simple con los diferentes grados de LARS Score. Como objetivos secundarios buscaremos evidenciar cuáles son las dimensiones funcionales de la vida que más se ven afectadas.

\section{Materiales y Método}

Se trata de un estudio de tipo transversal. Los pacientes invitados a participar fueron buscados en la base de datos de la Unidad de Coloproctología del Hospital Clínico de la Universidad de Chile. Los criterios de inclusión corresponden a los siguientes: pacientes operados con intención curativa entre los años 2004-2017, tumores que se encontraran a $\leq 10$ $\mathrm{cm}$ del margen anal (recto medio-bajo), pacientes con tumores de recto bajo se definió como aquellos que se encontraban a $\leq 5 \mathrm{~cm}$ del margen anal; se incluyen también a los pacientes con o sin radioquimioterapia neoadyuvante, con más de doce meses desde el cierre de su ileostomía y aquellos que aceptaron responder la encuesta de forma presencial o telefónica. Los criterios de exclusión fueron: pacientes que presentaban metástasis al momento de la cirugía, pacientes a los cuales se les realizó una colectomía total con anastomosis íleo-rectal o panproctocolectomía con reservorio ileal; por último, se excluyeron aquellos que rechazaron la invitación a ser parte del trabajo o que fue imposible su ubicación.

Para la evaluación del LARS se utilizó LARS Score la cual se encuentra validada al español chileno ${ }^{10}$, dicho score mide 5 ítems; incontinencia a líquidos, a gases, frecuencia de deposiciones, fraccionamiento de la defecación y urgencia defecatoria. Para evaluar la calidad de vida se utilizó la encuesta EuroQoL-5 (EQ-5D) ${ }^{11}$ la cual se encuentra validada en Chile tanto como para su idioma como para el resultado de sus índices ${ }^{12,13}$, este cuestionario nos entrega la evaluación de 5 dimensiones: movilidad, cuidado personal, actividades habituales, dolor/ malestar, angustia/depresión; también nos entrega un índice de calidad de vida que tiene como rango $0-1$, siendo 1 una salud perfecta y 0 muerte (Figura 1). La aplicación de los cuestionarios se realizó en 


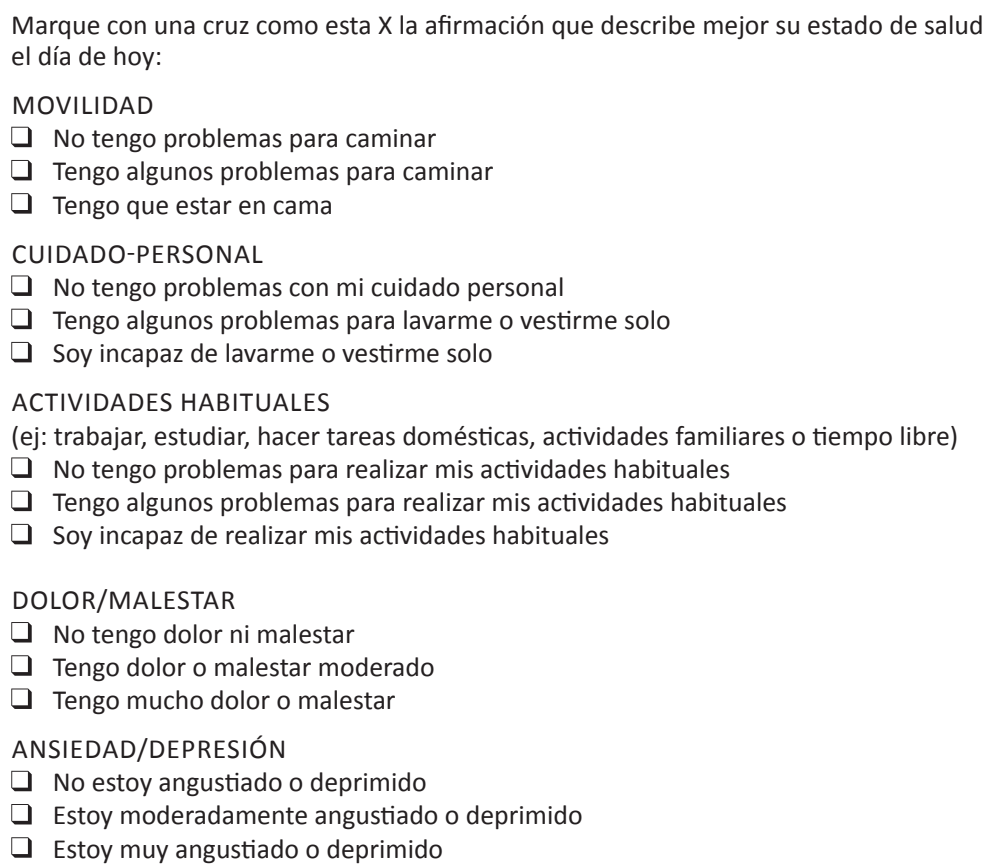

Figura 1. Encuesta EQ-5D y EQ-VAS (versión para Chile)

grupo por teléfono y otros de forma presencial. Por motivos metodológicos no se aplicó la escala visual análoga del EQ-5D, la cual solo se aplica de forma presencial.

Se realiza análisis demográfico de los pacientes y del tipo de cirugía. En el análisis exploratorio de datos se utilizaron estadísticos descriptivos expresados en porcentajes, promedios y desviaciones estándar. Para la determinación de asociaciones

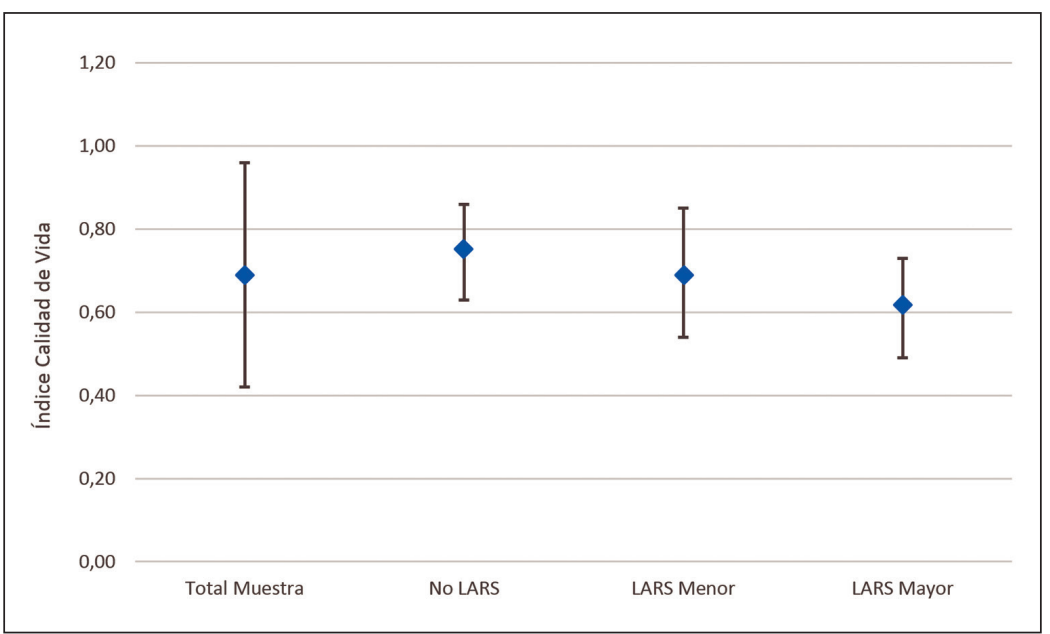

Figura 2. Índices promedio de calidad de vida y su relación con el nivel de LARS Score.
Tabla 1. Características generales del total de la serie

\begin{tabular}{|lc|}
\hline & $\mathbf{n}=\mathbf{5 4}$ \\
\hline Edad & $58,44(12,81)$ \\
\hline Menores de 65 años & $68,52 \%$ \\
Sexo (masculino) & $62,16 \%$ \\
Índice calidad de vida & $0,69(0,27)$ \\
\hline Altura del tumor & \\
Recto medio & $51,58 \%$ \\
Recto bajo & $48,15 \%$ \\
Tipo de cirugía & \\
RAB & $46,30 \%$ \\
RAUB & $31,48 \%$ \\
RAUBIE & $22,22 \%$ \\
Cirugía con TME & $53,70 \%$ \\
Cirugía laparoscópica & $57,41 \%$ \\
Radioterapia neoadyuvante & $83,33 \%$ \\
\hline Ileostomía protección & $100,00 \%$ \\
\hline Distribución de LARS & \\
Sin LARS & $40,74 \%$ \\
LARS Menor & $22,22 \%$ \\
LARS Mayor & $37,03 \%$ \\
LARS Menor y Mayor & $59,25 \%$ \\
\hline RAB: Resccón anterior baja & RAUB \\
\hline
\end{tabular}

RAB: Resección anterior baja; RAUB: Resección anterior ultra baja; RAUBIE: Resección anterior ultra baja interesfintérica; TME: Resección total del mesorrecto; LARS: Síndrome de resección anterior baja.

entre variables se utilizó $\chi^{2}$ de Pearson, $t$-test de Student y ANOVA según correspondiese. El análisis se realizó en el programa estadístico STATA 14.2. Se consideró estadísticamente significativo un valor de $\mathrm{p}<0,05$.

\section{Resultados}

Durante el periodo estudiado 54 pacientes contestaron la encuesta, que corresponde a un $56 \%$ de pacientes que cumplían los criterios de inclusión. Se observa un $62,16 \%$ de hombres y un promedio de edad 58,44 (DE 12,81). El 83,33\% de los pacientes recibió radioquimioterapia neoadyuvante, un $48,15 \%$ corresponden a tumores de recto bajo y un $53,70 \%$ corresponden a TME (Tabla 1 ). En la misma tabla observamos que el $37,03 \%$ presentó LARS Mayor y 59,25\% algún grado de LARS.

El índice de calidad de vida promedio de toda la serie es de un 0,69 (DE 0,27), sin diferencia significativa entre los promedios según LARS Score $(\mathrm{p}=0,246)$. En la Figura 2 se observa cómo el promedio de los índices de calidad de vida, disminuye 
Tabla 2. Características generales según LARS Score

\begin{tabular}{|lcccc|}
\hline & No LARS $(\mathbf{n}=\mathbf{2 2})$ & LARS Menor $(\mathbf{n}=\mathbf{1 2})$ & LARS Mayor $(\mathbf{n}=\mathbf{2 0})$ & Valor p \\
Edad & $55,63(12,31)$ & $61,00(12,54)$ & $60,00(13,52)$ & 0,408 \\
Menores de 65 años & $86,36 \%$ & $75,00 \%$ & $45,00 \%$ & 0,014 \\
Sexo (masculino) & $59,09 \%$ & $83,33 \%$ & $55,00 \%$ & 0,244 \\
Índice calidad de vida & $0,75(0,21)$ & $0,69(0,27)$ & $0,61(0,31)$ & 0,246 \\
Altura del tumor & & & & 0,029 \\
$\quad$ Recto medio & $50,00 \%$ & $83,33 \%$ & $35,00 \%$ & 0,065 \\
Recto bajo & $50,00 \%$ & $16,67 \%$ & $65,00 \%$ & \\
Tipo de cirugía & & & & \\
RAB & $45,45 \%$ & $75,00 \%$ & $50,00 \%$ & 0,047 \\
RAUB & $22,73 \%$ & $16,67 \%$ & $20,00 \%$ & 0,475 \\
RAUBIE & $31,82 \%$ & $8,33 \%$ & $70,00 \%$ & 0,528 \\
Cirugía con TME & $54,00 \%$ & $25,00 \%$ & $65,00 \%$ & $90,00 \%$ \\
Cirugía laparoscópica & $54,55 \%$ & $50,00 \%$ & $75,00 \%$ & \\
Radioterapia neoadyuvante & $81,82 \%$ & & & \\
\hline
\end{tabular}

RAB: Resección anterior baja; RAUB: Resección anterior ultra baja; RAUBIE: Resección anterior ultra baja interesfintérica; TME: Resección total del mesorrecto.

a medida que empeora el LARS Score. El índice de calidad de vida del LARS Mayor es de 0,61 (DE 0,31 ). Existe una diferencia de 14 puntos entre los promedios de No LARS y LARS Mayor (Tabla 2).

Al analizar los grupos etarios, los menores de 65 años, son el grupo con mayor porcentaje de No LARS y LARS Menor, lo cual es estadísticamente significativo. En los pacientes con LARS Menor el $83,33 \%$ presentaron tumores en recto medio y el $65 \%$ de los LARS Mayor presentaron tumores bajos, existiendo diferencia significativa. El $70 \%$ de los pacientes con LARS Mayor presentaron resecciones totales del mesorrecto, RAUB y RAUBIE, lo cual es estadísticamente significativo (Tabla 2).

La Tabla 3 nos muestra que, en las dos primeras dimensiones analizadas, movilidad y cuidado personal, ningún encuestado presenta alteraciones severas. Al analizar el resto de las dimensiones, encontramos que el $46,3 \%$ presenta algún grado de problema para realizar sus actividades habituales, existiendo un $7,41 \%$ que es incapaz de realizar sus actividades habituales.

En el análisis de la Tabla 4, al comparar las dimensiones afectadas con el nivel de LARS Score, encontramos que en el grupo de LARS Menor el $66,67 \%$ presenta alguna alteración en sus actividades habituales. Los pacientes con LARS Mayor, el $45 \%$ presenta algún grado de dolor o malestar y el $55 \%$ se encuentra angustiado o deprimido, este último con una diferencia significativa.
Tabla 3. Distribución según dimensiones de calidad de vida afectado

\begin{tabular}{|lr|}
\hline Dimensiones de calidad de vida & $\mathbf{n}=\mathbf{5 4}$ \\
Movilidad & \\
Sin problemas para caminar & $79,63 \%$ \\
Algunos problemas para caminar & $20,37 \%$ \\
Debo estar en cama & $0,00 \%$ \\
Porcentajes de pacientes con cualquier tipo de problema en movilidad & $20,37 \%$ \\
Cuidado personal & \\
Sin problemas con el cuidado personal & $66,67 \%$ \\
Algunos problemas para lavarme y vestirme solo & $33,33 \%$ \\
Incapaz de lavarme y vestirme solo & $0,00 \%$ \\
Porcentaje de pacientes con cualquier tipo de problema en cuidado personal & $33,33 \%$ \\
Actividades habituales & \\
Sin problemas para realizar actividades & $53,70 \%$ \\
Algunos problemas para realizar actividades & $38,89 \%$ \\
Incapaz de realizar las actividades & $7,41 \%$ \\
Porcentajes de pacientes con cualquier nivel de problema en actividades & \\
habituales & $46,30 \%$ \\
Dolor/malestar & \\
Sin dolor ni malestar & $72,22 \%$ \\
Tengo dolor o malestar moderado & $20,37 \%$ \\
Tengo mucho dolor o malestar & $4,00 \%$ \\
Porcentaje de pacientes con algún nivel de dolor/malestar & $24,37 \%$ \\
Angustia/depresión & \\
Sin angustia o depresión & $66,67 \%$ \\
Moderadamente angustiado o deprimido & $29,63 \%$ \\
Muy angustiado o deprimido & $3,70 \%$ \\
Porcentajes de pacientes con alteración de angustia/depresión & $33,33 \%$ \\
\hline
\end{tabular}


Tabla 4. Dimensiones de calidad de vida según LARS Score

\begin{tabular}{|c|c|c|c|c|}
\hline Dimensiones de calidad de vida & $\begin{array}{c}\text { No LARS } \\
(n=22)\end{array}$ & $\begin{array}{l}\text { LARS Menor } \\
\quad(\mathrm{n}=12)\end{array}$ & $\begin{array}{l}\text { LARS Mayor } \\
\quad(\mathbf{n}=\mathbf{2 0})\end{array}$ & Valor $\mathbf{p}$ \\
\hline Movilidad & & & & 0,745 \\
\hline Sin problemas para caminar & $77,27 \%$ & $75,00 \%$ & $85,00 \%$ & \\
\hline Debo estar en cama & $0,00 \%$ & $0,00 \%$ & $0,00 \%$ & \\
\hline Porcentajes de pacientes con cualquier tipo de problema en movilidad & $22,73 \%$ & $25,00 \%$ & $15,00 \%$ & 0,745 \\
\hline Algunos problemas para lavarme y vestirme solo & $31,82 \%$ & $41,67 \%$ & $30,00 \%$ & \\
\hline Incapaz de lavarme y vestirme solo & $0,00 \%$ & $0,00 \%$ & $0,00 \%$ & \\
\hline Porcentaje de pacientes con cualquier tipo de problema en cuidado personal & $31,82 \%$ & $41,67 \%$ & $30,00 \%$ & 0,780 \\
\hline Actividades habituales & & & & 0,434 \\
\hline Sin problemas para realizar actividades & $63,64 \%$ & $33,33 \%$ & $55,00 \%$ & \\
\hline Sin dolor ni malestar & $81,82 \%$ & $83,33 \%$ & $55,00 \%$ & \\
\hline Tengo dolor o malestar moderado & $18,18 \%$ & $16,67 \%$ & $25,00 \%$ & \\
\hline Tengo mucho dolor o malestar & $0,00 \%$ & $0,00 \%$ & $20,00 \%$ & \\
\hline Porcentaje de pacientes con algún nivel de dolor/malestar & $18,18 \%$ & $16,67 \%$ & $45,00 \%$ & 0,095 \\
\hline Angustia/depresión & & & & 0,049 \\
\hline Sin angustia o depresión & $72,73 \%$ & $91,67 \%$ & $45,00 \%$ & \\
\hline Moderadamente angustiado o deprimido & $27,27 \%$ & $8,33 \%$ & $45,00 \%$ & \\
\hline Muy angustiado o deprimido & $0,00 \%$ & $0,00 \%$ & $10,00 \%$ & \\
\hline Porcentajes de pacientes con alteración de angustia/depresión & $27,27 \%$ & $8,33 \%$ & $55,00 \%$ & 0,019 \\
\hline
\end{tabular}

Tabla 5. Dimensiones de la calidad de vida y la presencia de LARS Mayor

\begin{tabular}{|c|c|c|c|c|}
\hline & $\begin{array}{l}\text { LARS Mayor } \\
\quad(n=20)\end{array}$ & Valor $\mathbf{p}$ & Odds Ratio & IC $95 \%$ \\
\hline Dolor/malestar & & 0,015 & & \\
\hline Sin dolor ni malestar & $55,00 \%$ & & & \\
\hline Tengo mucho dolor o malestar & $20,00 \%$ & & & \\
\hline Porcentaje de pacientes con algún nivel de dolor/malestar & $45,00 \%$ & 0,030 & 3,8 & $1,09-13,27$ \\
\hline Moderadamente angustiado o deprimido & $45,00 \%$ & & & \\
\hline Muy angustiado o deprimido & $10,00 \%$ & & & \\
\hline Porcentajes de pacientes con alteración de angustia/depresión & $55,00 \%$ & 0,010 & 4,7 & $1,40-15,82$ \\
\hline
\end{tabular}

Al analizar solo el grupo de LARS Mayor comparado con el resto, encontramos que existen dos dimensiones en donde hay diferencias estadísticamente significativas, dolor/malestar y angustia/ depresión, presentando un odds ratio de 3,8 para el primero y 4,7 para el segundo (Tabla 5).

\section{Discusión}

Nuestro estudio es el primero en buscar la asociación entre la calidad de vida y LARS Score, utilizando el cuestionario EQ-5D. Emmertsen et al. ${ }^{5}$, en su publicación pionera de 2012, donde valida el 
cuestionario LARS Score con la calidad de vida, no utiliza un parámetro objetivo en la evaluación de esta última, como los cuestionarios EQ-5D o EORTC-30, sin embargo, posteriormente diferentes autores validaron el LARS Score con el EORT$30^{7,8,14,15}$

Nuestro estudio nos revela que no existe una diferencia estadísticamente significativa entre los índices de calidad de vida y el nivel de LARS, sin embargo, encontramos una tendencia clara, que nos indica menor índice de calidad de vida, a medida que empeora el LARS Score. El que no encontremos diferencia significativa puede estar determinado por el bajo $\mathrm{n}$ de nuestra muestra. Al utilizar el EORTC-30 para cuantificar la calidad de vida, algunos autores hacen énfasis en que debe existir una diferencia de más de 10 puntos entre los índices para considerar clínicamente significativa la diferencia, independiente del valor $\mathrm{p}^{7}$. Si bien nosotros utilizamos otro cuestionario, encontramos una diferencia de 14 puntos entre el índice de calidad de No LARS y LARS Mayor, lo cual podría ser relevante.

Nuestra serie presenta una prevalencia de un 37,03\% de LARS Mayor, la cual se encuentra dentro de lo publicado en series internacionales. Croese et al en su metaanálisis publicado el 2018, nos evidencian que la prevalencia de LARS Mayor variaba entre $17,8 \%$ y $56 \%$, calculando una prevalencia pro-

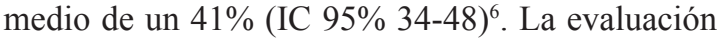
del LARS Score luego de 12 meses de ser operado o del cierre de la ileostomía es fundamental, ya que de esa manera se objetiva realmente el deterioro de su función intestinal, considerando que con el tiempo un $10 \%$ puede empeorar y un $14 \%$ mejorar $^{16}$.

En el análisis de las dimensiones afectadas encontramos alteraciones significativas en lo relacionado con dolor/malestar y angustia/depresión. Los pacientes con LARS Mayor presentan 3,8 veces más posibilidades de desarrollar algún dolor/malestar que el resto de los pacientes. El dolor se encuentra en el tercer lugar de síntomas con mayor prevalencia en los pacientes que se les aplicó EORTC-30, en conjunto con diarrea e insomnio, encontrando una diferencia significativa al comparar el dolor según el nivel de LARS Score ${ }^{7,8,16,17}$. Es relevante tener este síntoma en consideración, debido a que pueden desarrollar algún grado de dolor crónico, el cual no se encuentra bien investigado en los pacientes operados por cáncer de recto. Emmertsen et al. ${ }^{15}$ publica que luego de 12 meses el 9,9\% de los pacientes experimentan dolor abdomianl más de una vez por semana, y un 9,5\% refiere dolor al defecar, dichos dolores se encuentran relacionados de forma significativa con el nivel de LARS Score.
La presencia de LARS Mayor confiere 4,7 veces más probabilidades de desarrollar algún grado de angustia o depresión. Los resultados publicados luego de la aplicación del EORTC-30 evidencian que, las alteraciones en la esfera emocional se encuentran dentro de las primeras tres dimensiones más afectadas, en conjunto con las alteraciones de las actividades sociales y cotidianas ${ }^{14,18}$. Es vital tener en consideración los trastornos emocionales, para poder entregar un apoyo multidisciplinario a los pacientes operados por cáncer de recto, en especial al grupo con LARS Mayor.

El 46\% de los pacientes presentan algún grado de alteración en sus actividades habituales, si bien no existe diferencia significativa entre los niveles de LARS, los pacientes con LARS Menor y Mayor presentan una mayor prevalencia de esta dimensión funcional. Lo anterior se puede explicar por el bajo $\mathrm{n}$ y por lo abierta de las preguntas. En los resultados publicados, luego de aplicar el cuestionario EORTC-30, los problemas en las funciones cotidianas y sociales, son lo más frecuente, y se relacionan directamente con el nivel de LARS Score ${ }^{7,14,16,18}$.

Si bien, dentro de los objetivos no se encontraba la búsqueda de factores demográficos y/o quirúrgicos relacionados con el nivel de LARS Score, evidenciamos que los pacientes con LARS Mayor en su mayoría tuvieron TME y son mayores de 65 años, a pesar que los promedios de edad son similares en los tres grupos de LARS Score. La TME es uno de los factores de mayor impacto en el desarrollo de LARS, en conjunto con la radioquimioterapia neoadyuvante ${ }^{19-21}$. Los menores de 65 años han reportado una mayor prevalencia de disfunción defecatoria y se ha considerado como factor de riesgo para el desarrollo de LARS, la explicación para lo anterior es la selección de casos para cirugías con preservación de esfínter y la dismotilidad colónica que ocurre con el envejecimiento que genera reducción de la urgencia y frecuencia defecatoria ${ }^{16,19,20}$. Battersby et al. ${ }^{19}$, en su estudio restrospectivo donde valida POLARS Score (Pre-Operative LARS Score), expone que esta asociación con la edad debe ser estudiada a mayor profundidad en futuras publicaciones. Otro factor a considerar es que en las grandes series mencionan como sesgo que el grupo menor de 65 años fue quien respondió en mayor porcentaje los cuestionarios. Como contraparte existen publicaciones que no relacionan la edad con la función defecatoria luego de la resección por cáncer ${ }^{21,22}$, incluso algunos estudios demuestran una fuerte asociación entre disfunción defecatoria y el envejecimiento ${ }^{24}$.

La aplicación de los cuestionarios de calidad de vida, LARS Score y actualmente POLARS Score 
debieran ser herramientas de uso habitual en nuestra práctica clínica, entregándonos información objetiva sobre aspectos funcionales de la vida del paciente, que muchas veces no lo consideramos, ya que nos centramos principalmente en lo oncológico. El uso de estas herramientas, para futuras publicaciones, deberían ser de forma prospectiva, con el fin de disminuir sesgos y conocer el estado defecatorio basal de los pacientes antes de comenzar el tratamiento, considerando que, en la población general, entre los 50 y 79 años, el 18,8\% de las mujeres y el 9,6\% de los hombres presentan puntajes $\geq 30$ en LARS Score $^{25}$.

El artículo presenta la falencia de no ser prospectivo y tener un sesgo de selección al incluir un grupo de pacientes con cáncer de recto muy específico. En futuros trabajos debemos ampliar nuestros criterios de inclusión y trabajar dirigidamente en los factores relacionados con el desarrollo de LARS.

\section{Conclusión}

El LARS Score se relaciona de manera no significativa con el índice de calidad de vida entregado por el cuestionario EQ-5D. Se observa una tendencia, en donde el índice de calidad de vida disminuye a medida que empeora el nivel de LARS. Las dimensiones de calidad de vida más afectadas son las relacionadas con las actividades habituales, dolor/ malestar y angustia/depresión, estas dos últimas tienen mayor posibilidad de manifestarse si el paciente presenta LARS Mayor. El cuestionario EQ-5D es una herramienta fácil de aplicar en la evaluación de calidad de vida.

\section{Responsabilidades éticas}

Protección de personas y animales. Los autores declaran que para esta investigación no se han realizado experimentos en seres humanos ni en animales.

Confidencialidad de los datos. Los autores declaran que en este artículo no aparecen datos de pacientes.

Conflictos de interés: no hay.

\section{Bibliografía}

1. Siegel RL, Fedewa SA, Anderson WF, Miller KD, Ma J, Rosenberg PS, et al. Colorectal Cancer Incidence Patterns in the United States, 1974-2013. J Natl Cancer Inst. 2017;109:322-28.

2. Heald RJ, Ryall RD. Recurrence and survival after total mesorectal excision for rectal cancer. Lancet 1986;1(8496):147982.

3. Rödel C, Graeven U, Fietkau R, Hohenberger W, Hothorn T, Arnold D, et al. Oxaliplatin added to fluorouracilbased preoperative chemoradiotherapy and postoperative chemotherapy of locally advanced rectal cancer (the German $\mathrm{CAO} /$ ARO/AIO-04 study): final results of the multicentre, open-label, randomised, phase 3 trial. Lancet Oncol. 2015;16:979-89.

4. Sauer R, Liersch T, Merkel S, Fietkau $\mathrm{R}$, Hohenberger W, Hess C, et al. Preoperative versus postoperative chemoradiotherapy for locally advanced rectal cancer: results of the German $\mathrm{CAO} /$ ARO/AIO-94 randomized phase III trial after a median follow-up of 11 years. J Clin Oncol. 2012;30(16):1926e33. https://
doi.org/10.1200/JCO.2011.40.1836.

5. Emmertsen KJ, Laurberg S. Low anterior resection syndrome score: development and validation of a symptom-based scoring system for bowel dysfunction after low anterior resection for rectal cancer. Ann Surg. 2012;255:922-28.

6. Croese AD, Lonie JM, Trollope AF, Vangaveti VN, Ho YH. A meta-analysis of the prevalence of Low Anterior Resection Syndrome and systematic review of risk factors. Int J Surg. 2018;56:234-41.

7. Juul T, Ahlberg M, Biondo S, Espin E, Jimenez LM, Matzel KE, et al. Low anterior resection syndrome and quality of life: an international multicenter study. Dis Colon Rectum. 2014;57(5):585-91.

8. Battersby NJ, Juul T, Christensen P, Janjua AZ, Branagan G, Emmertsen $\mathrm{KJ}$, et al. Predicting the Risk of BowelRelated Quality-of-Life Impairment After Restorative Resection for Rectal Cancer: A Multicenter Cross-Sectional Study. Dis Colon Rectum 2016;59:270-80.

9. Fayers P, Bottomley A. Quality of life research within the EORTC-the EORTC QLQ-C30. European Organisation for Research and Treatment of Cancer. Eur J
Cancer 2002;38:S125-33.

10. López S, Carrillo K, Sanguineti A, Azolas R, Díaz M, Bocic G, et al. Adaptación transcultural del cuestionario acerca de la función intestinal (LARS Score) para su aplicación en pacientes operados de cáncer de recto medio y bajo. Rev Chil Cir. 2017;69:44-8.

11. EuroQol Research Foundation. EQ-5D-3L User Guide, 2018. Available from: https:// euroqol.org/publications/user-guides.

12. Zarate V, Kind P, Valenzuela P, Vignau A, Olivares-Tirado P, Muñoz A. Social valuation of EQ-5D health states: the Chilean case. Value Health 2011;14:113541.

13. Olivares-Tirado P. Calidad de vida relacionada a la salud (HR-QoL) en población general de Chile 2005. Chile: Departamento de Estudios y Desarrollo, Superintendencia de Salud, Gobierno de Chile 2006. Available from: http://www. supersalud.gob.cl/documentacion/666/ articles-3818_recurso_1.pdf

14. Trenti L, Gálvez A, Biondo S, Solís A, Vallribera-Valls F, Espin-Basany E, et al. Quality of life and anterior resection syndrome after surgery for mid to low 
ARTíCULO ORIGINAL

rectal cancer: A cross-sectional study. Eur J Surg Oncol. 2018;44:1031-39.

15. Emmertsen KJ, Laurberg S. Impact of bowel dysfunction on quality of life after sphincter-preserving resection for rectal cancer. Br J Surg. 2013;100:1377-87.

16. Pieniowski EHA, Palmer GJ, Juul T, Lagergren P, Johar A, Emmertsen KJ, et al. Low Anterior Resection Syndrome and Quality of Life After Sphincter-Sparing Rectal Cancer Surgery: A Long-term Longitudinal Follow-up. Dis Colon Rectum. 2019;62:14-20.

17. Engel J, Kerr J, Schlesinger-Raab A, Eckel R, Sauer H, Hölzel D. Quality of life in rectal cancer patients: a fouryear prospective study. Ann Surg. 2003;238:203-13.

18. Jiménez-Gómez LM, Espin-Basany E, Trenti L, Martí-Gallostra M, SánchezGarcía JL, Vallribera-Valls F, et al. Factors associated with low anterior resection syndrome after surgical treatment of rectal cancer. Colorectal Dis. 2017. Epub ahead of print. doi: 10.1111/codi.13901.

19. Battersby NJ, Bouliotis G, Emmertsen KJ, Juul T, Glynne-Jones R, Branagan G, et al. Development and external validation of a nomogram and online tool to predict bowel dysfunction following restorative rectal cancer resection: the POLARS score. Gut. 2018;67:688-96.

20. Bregendahl S, Emmertsen KJ, Lous J, Laurberg S. Bowel dysfunction after low anterior resection with and without neoadjuvant therapy for rectal cancer: a population-based cross-sectional study. Colorectal Dis. 2013;15:1130-39.

21. Lange MM, den Dulk M, Bossema ER, Maas CP, Peeters KC, Rutten HJ, et al. Risk factors for faecal incontinence after rectal cancer treatment. Br J Surg.
2007;94:1278-84.

22. Ho P, Law WL, Chan SC, Lam CK, Chu KW. Functional outcome following low anterior resection with total mesorectal excision in the elderly. Int J Colorectal Dis. 2003;18:230-33.

23. Perry $\mathrm{S}$, Shaw $\mathrm{C}$, McGrother $\mathrm{C}$, Matthews RJ, Assassa RP, Dallosso H, et al. Prevalence of faecal incontinence in adults aged 40 years or more living in the community. Gut. 2002;50:480-84.

24. MacLennan AH, Taylor AW, Wilson DH, Wilson D. The prevalence of pelvic floor disorders and their relationship to gender, age, parity and mode of delivery. BJOG. 2000;107:1460-70.

25. Juul T, Elfeki H, Christensen P, Laurberg S, Emmertsen KJ, Bager P. Normative Data for the Low Anterior Resection Syndrome Score (LARS Score). Ann Surg. 2019;269:1124-28. 